\title{
A vigilance task sensitive to the effects of stimulants, hypnotics, and environmental stress: The Scanning Visual Vigilance Test
}

\author{
HARRIS R. LIEBERMAN, BRYAN COFFEY, and JOHN KOBRICK \\ U.S. Army Research Institute of Environmental Medicine, Natick, Massachusetts
}

\begin{abstract}
The Scanning Visual Vigilance Test is a variable-length detection test designed to assess the ability of individuals to maintain visual alertness for sustained periods of time. The test was designed to be sensitive to changes in vigilance produced by subtle variations in performance, such as those produced by low doses of centrally acting food constituents, drugs, or environmental stress. The test has been shown to be sensitive to the effects of stimulants and sedatives, as well as cold stress and sleep loss. It requires the subject to continuously scan a video monitor to detect the occurrence of infrequent stimuli that are difficult to detect. The number of stimuli correctly detected, false alarms, and reaction times are recorded. The stimulus is a small rectangle displayed for $2 \mathrm{sec}$ at random locations on a darker region of a video monitor at random or pseudo-random times. The brightness of the stimulus can be adjusted for each subject individually on an automated threshold detection test. Training and test session length are defined by the experimenter. Hardware requirements are an IBM-compatible personal computer (286 or higher) with a color or grayscale VGA monitor.
\end{abstract}

Vigilance is a sensitive indicator of functional capability. It reflects the ability of individuals to process relevant information and respond in a timely fashion. Recent reports have provided detailed information on the mechanisms underlying vigilance performance and the various tasks that have been used to assess the underlying process or processes (Hirshkowitz, De La Cueva, \& Herman, 1993; Koelega, 1989; Tiplady, 1992). The assessment of vigilance is of considerable practical significance, since many activities, such as the operation of vehicles and industrial equipment, require maintenance of vigilance for long periods of time (Mackie, 1987). A number of accidents, such as the Three Mile Island nuclear reactor failure and commercial aircraft crashes, have been attributed, at least in part, to the failure of human operators to detect critical stimuli (Mitler, 1988; Office of Technology Assessment, 1991). It has also been suggested that vigilance tasks may be more relevant to the performance of everyday activities than the shorter tests of cognitive performance often employed in test batteries (Koelega, 1989).

The views, opinions, and/or findings in this report are those of the authors, and should not be construed as an official Department of the Army position, policy or decision, unless so designated by other official documentation. Human subjects participated in these studies after giving their free and informed voluntary consent. Investigators adhered to AR 70-25 and USAMRDC Regulation 70-25 on the use of volunteers in research. Citations of commercial organizations and trade names in this report do not constitute an official Department of the Army endorsement or approval of the products or services of these organizations. Correspondence should be addressed to H. R. Lieberman, Military Nutrition and Biochemisty Division, USARIEM (MCMR-UENBD), Natick, MA 01760-5007 (e-mail: hlieberman@natick-ccmail. army.mil)
One frequently employed vigilance test is the Wilkinson Auditory Vigilance Test (Wilkinson, 1968). This task has been used widely, because it is sensitive to a variety of independent variables and is relatively simple to administer (Ogilvie \& Broughton, 1992). In its original version, a tone was presented every $2 \mathrm{sec}$ for a period of $1 \mathrm{~h}$. White noise was presented in the background to mask extraneous stimuli. The background tones presented every $2 \mathrm{sec}$ were $500 \mathrm{msec}$ in duration. Randomly dispersed among these tones were 40 test tones that were perceptibly shorter. The subject's task was to detect the shorter test tones by responding before the next tone was presented. This test has been used in studies of sleep deprivation (Wilkinson, 1968) circadian rhythms (Craig, Wilkinson, \& Colquhoun, 1981), bright light (Dollins, Lynch, Wurtman, Deng, \& Lieberman, 1993), drugs (Koelega, 1989), hormones such as melatonin (Dollins, Lynch, Deng, et al., 1993), and food constituents (Clubley, Bye, Henson, Peck, \& Riddington, 1979; Lieberman, Wurtman, Garfield, Roberts, \& Coviella, 1987), as well as other factors.

Another task that has been used to evaluate vigilance is the Continuous Attention Task (CAT; Tiplady, 1992). For this test, subjects view a series of $3 \times 3$ patterns of squares on a monitor screen and respond when two successive patterns are identical. Patterns are displayed for $100 \mathrm{msec}$ at intervals of $1.5-2.5 \mathrm{sec}$. Yet another task is the Multiple Vigilance Test (MVT; Hirshkowitz et al., 1993), which requires the subject to continuously view a computer screen on which a masking stimuli is displayed. Every $4-11 \mathrm{sec}$ either a target or a nontarget pattern is displayed in place of the mask for $250 \mathrm{msec}$. The subject responds when a target pattern is presented. The target and the nontarget patterns differ from each other by being 
rotated $90^{\circ}$ from one another. The CAT requires the subject to continuously store and recall simple patterns from memory, making the test partly a memory task and partly a vigilance task. In addition, subjects may also actively seek out specific features to aid in their memory task. The MVT is a much simpler task for the subject, but it still requires continuous recall of the target pattern. Since the target and the nontarget are very similar, a subject could, over the period of a long test, occasionally reverse the categorization between target and nontarget stimuli.

It has been suggested that the main attributes of the most sensitive vigilance tasks, including the Wilkinson task, which render them sensitive to subtle variations in vigilance performance are long duration, infrequent occurrence of test stimuli, and monotony (Fine et al., 1994; Lieberman, 1989; Wilkinson, 1992). Recently, it has been reported that certain types of brief tasks can also be sensitive to small variations in alertness, particularly when sophisticated data analysis procedures are employed (Dinges, 1992). The Scanning Visual Vigilance Test (SVT), described in this article, was designed to include the apparently critical aspects of the Wilkinson Vigilance Test and other tests of vigilance that are also regarded as sensitive to treatment effects (Fine et al., 1994).

The SVT presents simple, near-threshold stimuli at pseudo-random locations on a microcomputer screen. To detect the stimuli, the subject must continuously scan the computer display. The test can run for up to $2 \mathrm{~h}$, with as many as 200 stimulus presentations permitted. As a result, changes in vigilance occurring over long periods of time can be continuously assessed. Another advantage of the SVT is that it assesses both the number of detections and reaction time. The test was designed to minimize the cognitive load, thereby emphasizing the vigilance aspects of the task.

In a recent study, we demonstrated that the SVT was sensitive to treatments that both enhance and impair performance (Figure 1; Fine et al., 1994). The SVT detected significant beneficial effects of a moderate dose of caffeine $(200 \mathrm{mg}$ ) roughly equivalent to two cups of coffee (Figure 1). A low dose of diphenhydramine $(25 \mathrm{mg})$, an over-the-counter antihistamine with sedative properties, impaired vigilance performance (Figure 1). Previously, similar effects of caffeine in this dose range have been detected on the Wilkinson Vigilance Task (Clubley et al., 1979; Lieberman et al., 1987). However, prior research suggested that such a low dose of diphenhydramine did not affect performance (Linnoila, 1973). The SVT has also been shown to be sensitive to the effects of environmental (cold) stress on subjects (Figure 2). Performance on the SVT decreased substantially after several hours in a cold chamber at $10^{\circ} \mathrm{C}$. Recently, we have also seen substantial changes in visual vigilance as assessed by the SVT in volunteers who were sleep deprived and exposed to environmental and exercise stress (Figure 3 ).

One criticism of the Wilkinson task is that it presents stimuli that are near the threshold of discrimination; thus it is difficult to test patients with impaired cognitive func- tion (Hirshkowitz et al., 1993). However, in a modification of the original Wilkinson task this potential problem was corrected by adjusting the difference between the test and background tones so that subjects detected approximately $50 \%$ of the stimuli in a pretest session (Lieberman et al., 1987). The SVT incorporates this modification of the Wilkinson task by assessing the initial detection threshold for each subject and presenting the test stimuli accordingly.

One disadvantage of the Wilkinson Vigilance Task is that it cannot be implemented on typical microcomputers used in laboratories without purchase or construction of additional equipment. The SVT, however, requires only a standard IBM-compatible personal computer. Also, it is suitable for field use since it will run on an IBMcompatible laptop computer without modification.

One potential disadvantage of the SVT is that it requires "active" scanning performance by the subject. To detect most stimuli, the subject must attempt to continuously scan the display rather than focus on a particular region of the screen. It has been suggested that automatic tasks are more sensitive to decrements in performance than tasks that require more active effort by the subject (Tiplady, 1992). Visual scanning is a fairly automatic process, however, with low cognitive load. Furthermore, many operational tasks with substantial vigilance components, both military and civilian, require visual scanning (Wiener, 1988). Examples of such tasks include scanning the sky or the cockpit controls while one is piloting aircraft, operating a power plant control room, monitoring a radar screen, and scanning the road during operation of a motor vehicle.

\section{TEST DESCRIPTION}

In the SVT, the subject views a computer monitor with either a dark or a light background intensity and is required to respond when a small rectangle $(2 \times 4$ pixels, $0.75 \times 1.85 \mathrm{~mm}$ ) of different intensity appears. Responses are made by pressing the space bar of the keyboard. The stimuli remain on the screen for $2 \mathrm{sec}$. A space bar press during this time is considered a hit. Failure to do so is scored a miss. Pressing the space bar at any other time counts as a false alarm response. The intensity of the stimulus can be preset by the experimenter, or a threshold test can be run to determine the ability of the subject to detect blocks of various intensities against a constant-intensity background. The background intensity, stimulus size, average delay between stimulus occurrences, and total test duration are determined by the experimenter before testing. A total of up to 64 brightness levels, depending on the capabilities of the monitor employed, from black to white are available for the background and stimulus levels.

The software system incorporates two utility programs. One is used to set up the visual vigilance task and pseudo-random timing/position files, and then to run a threshold test. The second program is used to analyze the output files. 
A

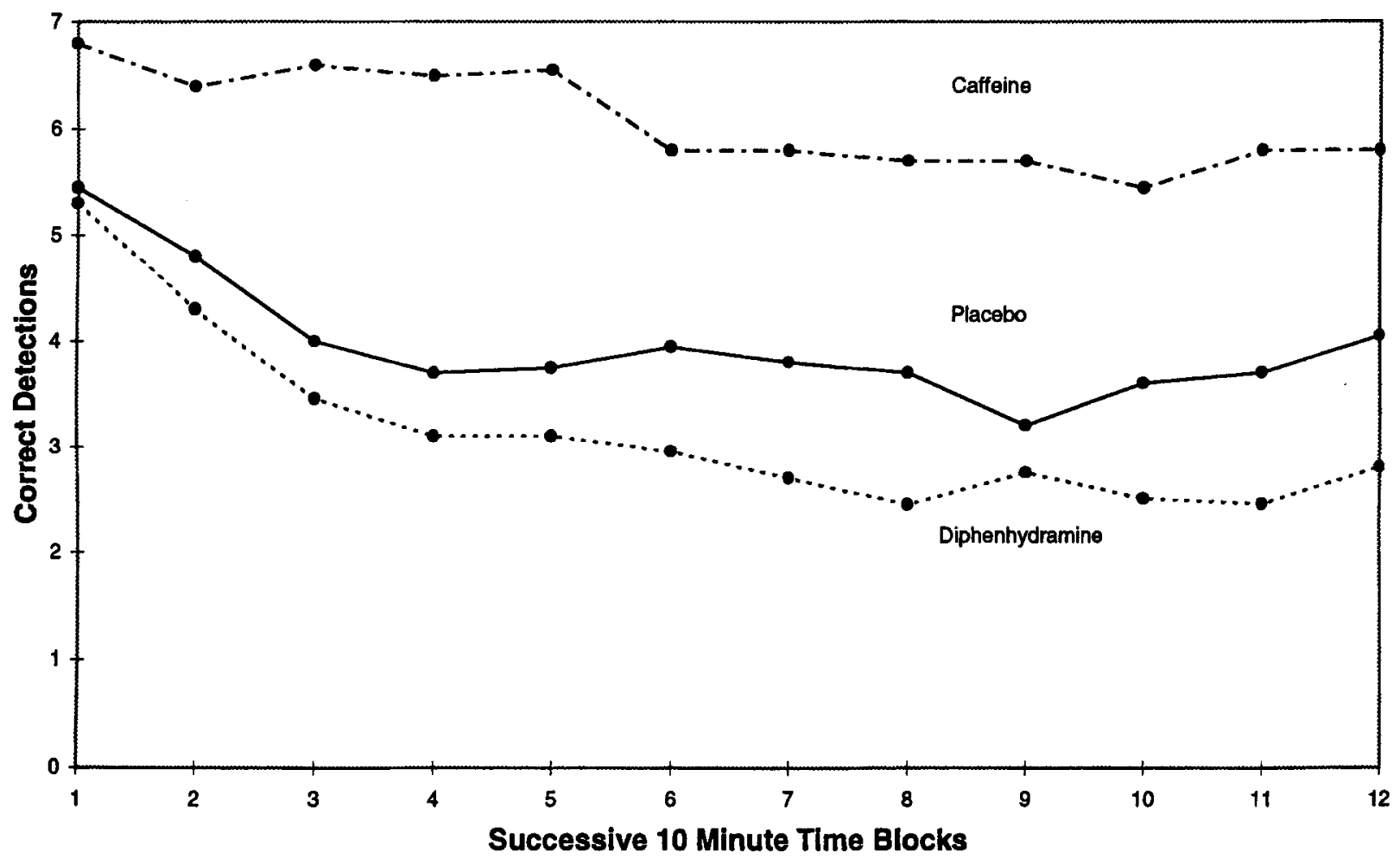

$\mathbf{B}$

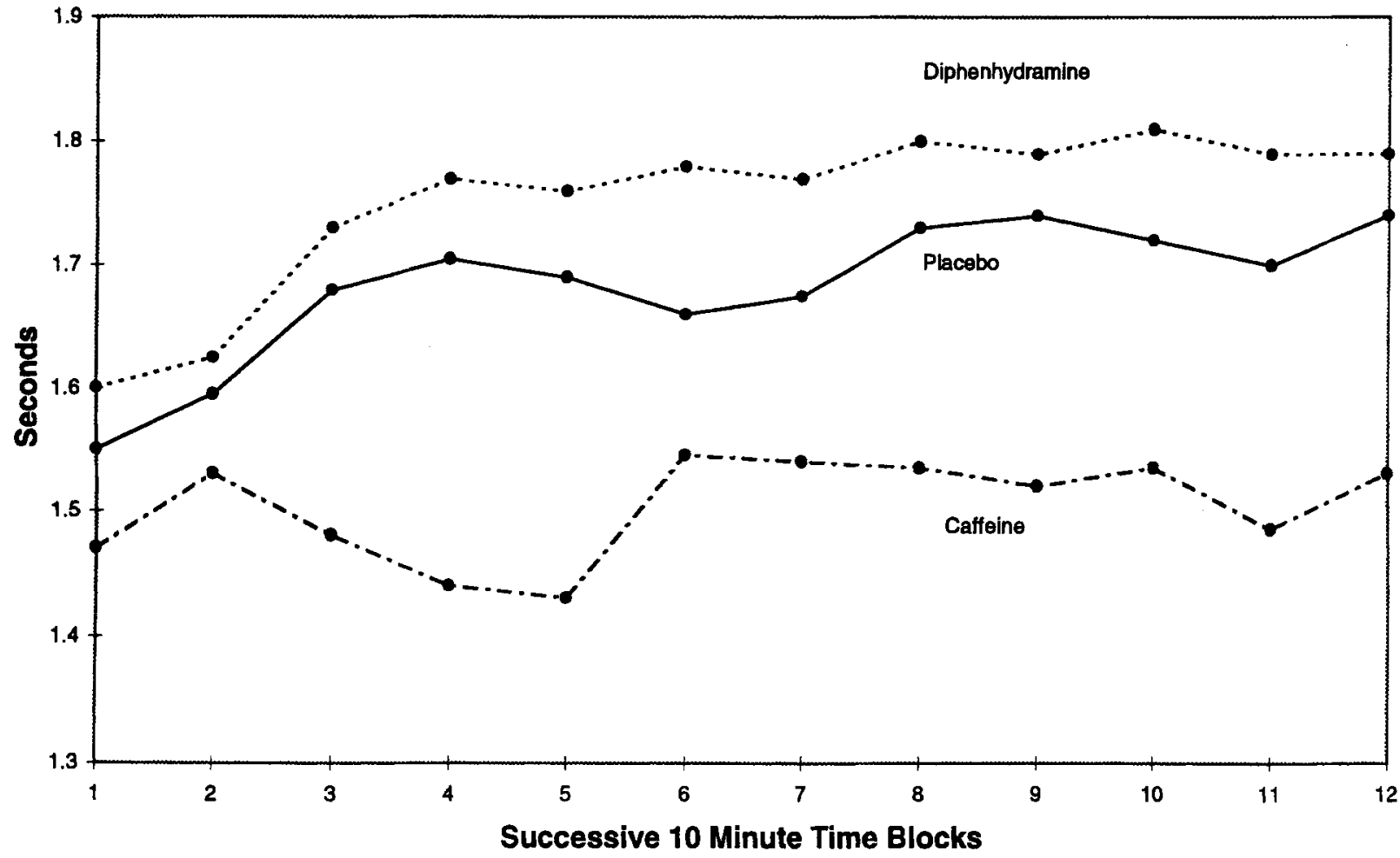

Figure 1. (A) Effects of caffeine (200 $\mathrm{mg}$ ) and diphenhydramine (25 $\mathrm{mg}$ ) on mean number of correct detections (hits) in the Scanning Visual Vigilance Task. Group mean detection frequency by drug treatment is plotted over successive 10 -min blocks; 10 test stimuli are presented per each block. (B) Effects of caffeine and diphenhydramine on reaction time. Group mean response time by drug treatment is plotted over successive 10 -min blocks. 


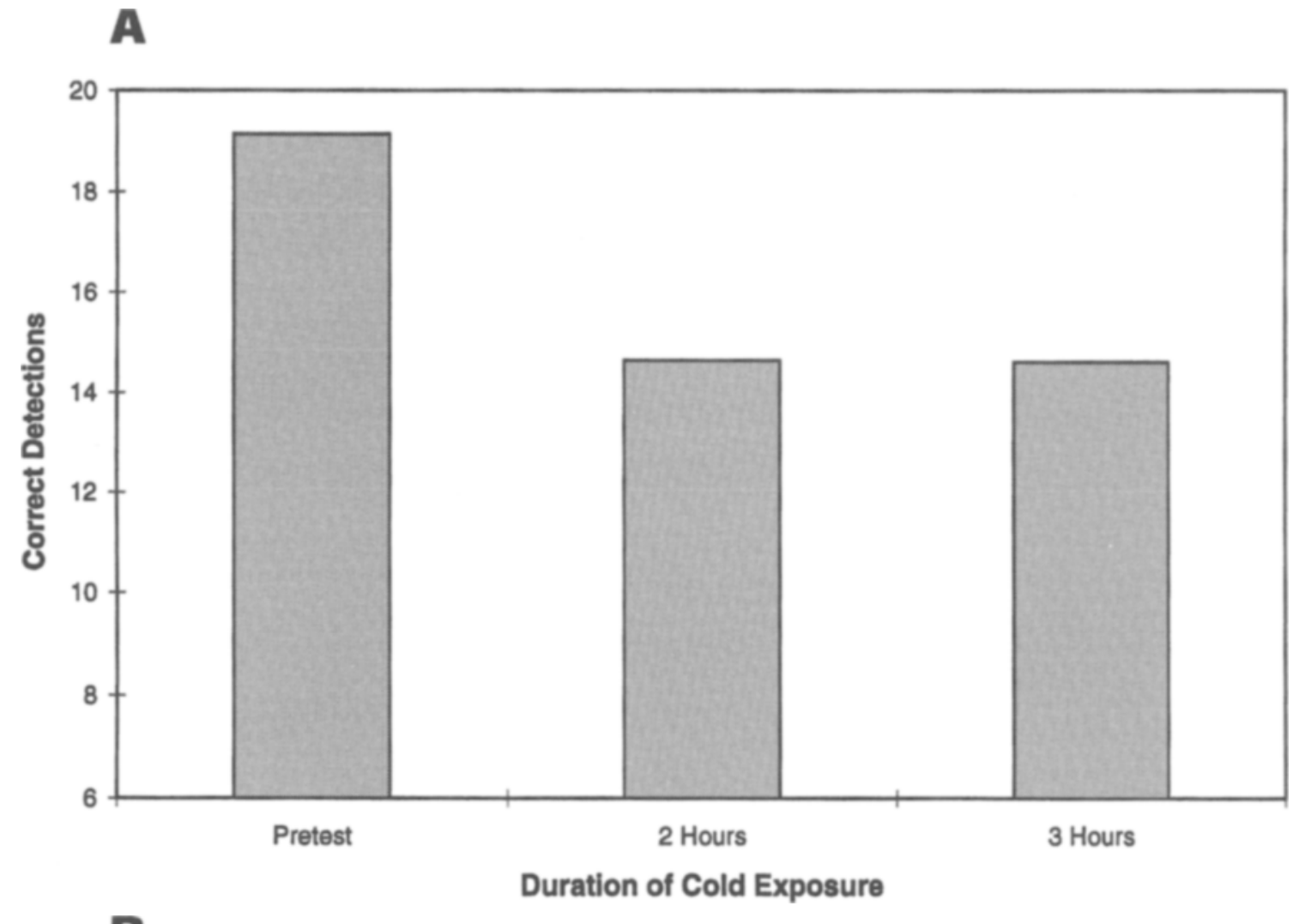

$\mathbf{B}$

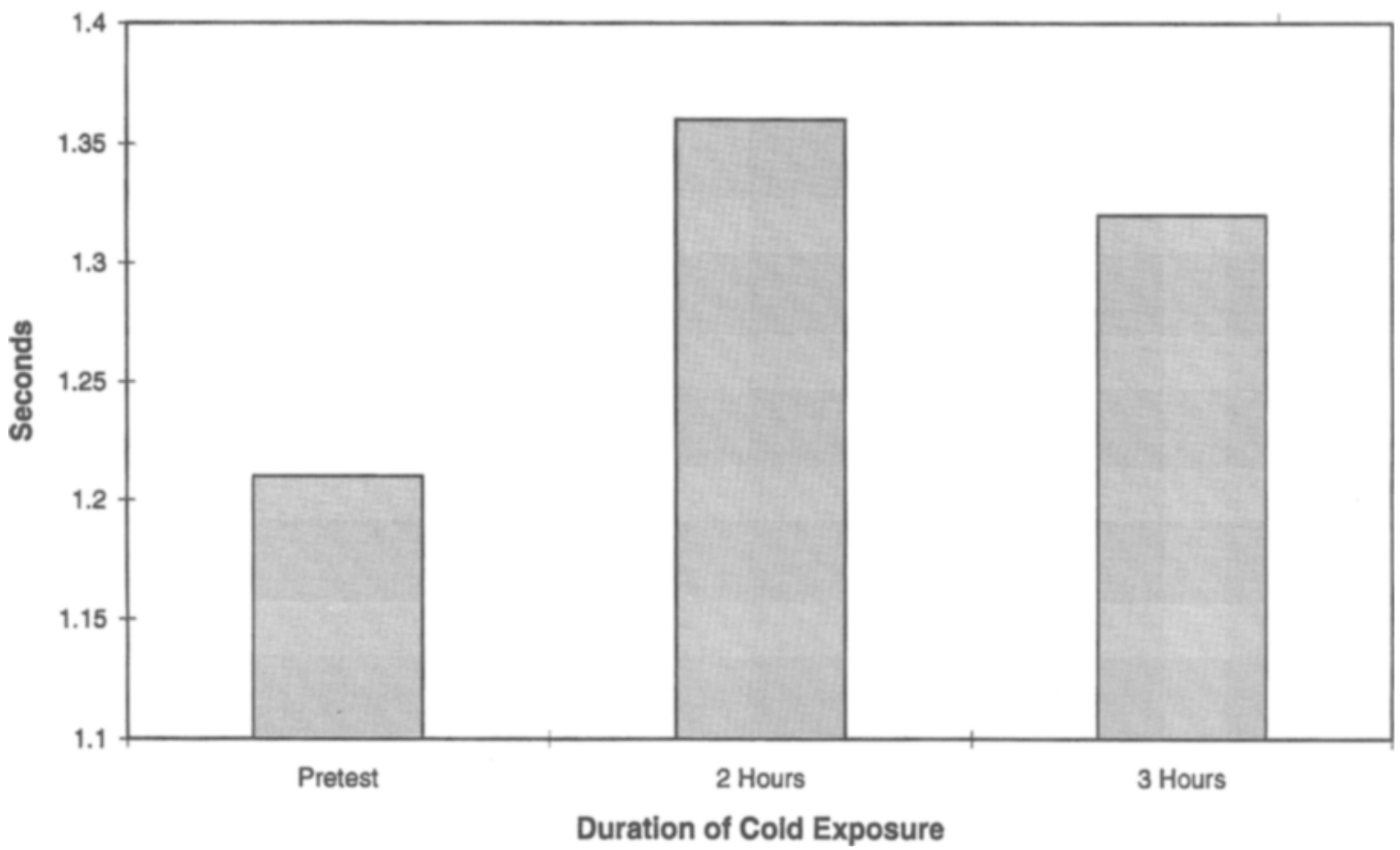

Figure 2. (A) Mean number of correct detections (hits) during acute exposure to cold for $3 \mathrm{~h}$. Subjects were exposed to $10^{\circ} \mathrm{C}$ cold air in an environmental chamber. Testing on the Scanning Visual Vigilance Task was conducted for 15 min every $2 \mathrm{~h}$. A total of 22 stimuli were presented during each 15-min test session. (B) Mean response time during acute exposure to cold for $3 \mathrm{~h}$. 


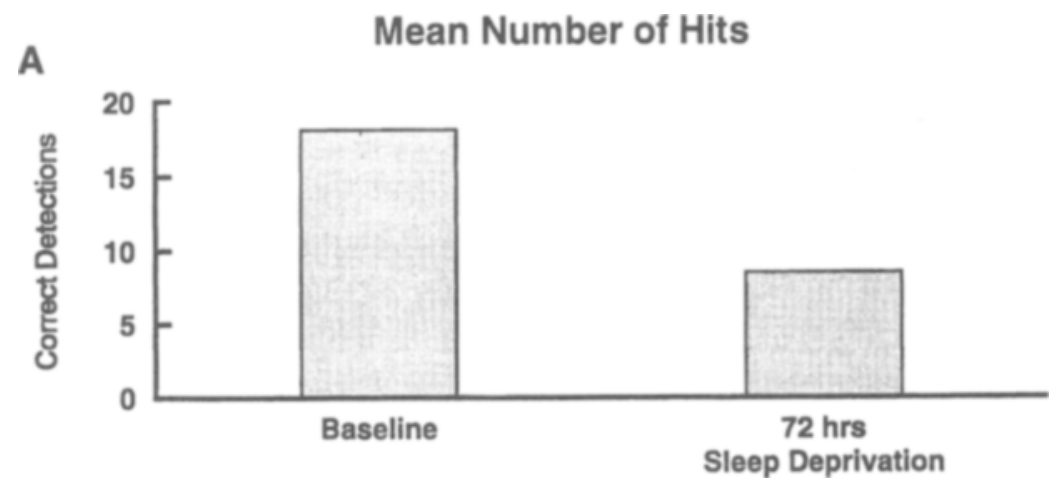

B Mean Response

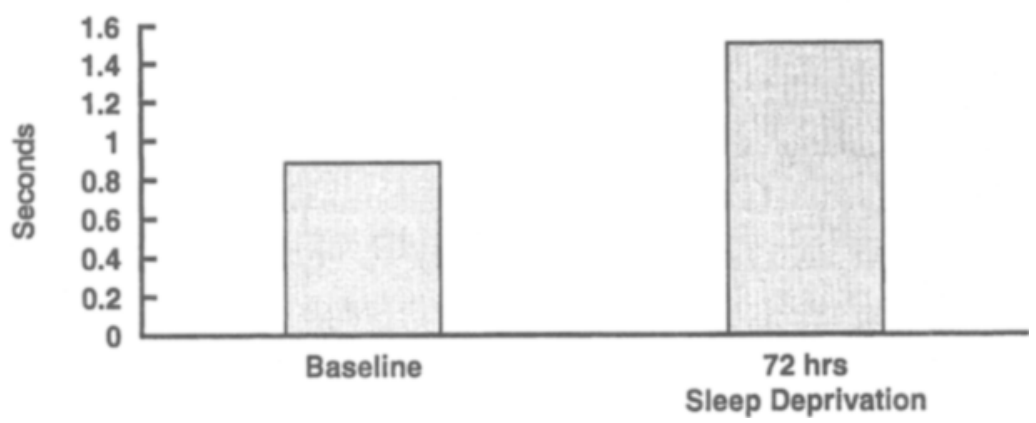

Figure 3. (A) Mean number of correct detections (hits) on the Scanning Visual Vigilance Task after $72 \mathrm{~h}$ of nearly total sleep deprivation in combination with physical and environmental stress. The test session was $16 \mathrm{~min}$ in duration, and 20 test stimuli were presented in that period. (B) Group mean response time on the SVT after 72 h of near total sleep deprivation in combination with physical and environmental stress.

This program is written in Borland $\mathrm{C}++$ Version 3.1 for IBM PCs and compatibles ( 286 or better). The program is an upgraded version of the SVT program previously developed at USARIEM using Microsoft Quick Basic Version 4.00b (Fine et al., 1994). Either a color or a grayscale VGA display can be employed for stimulus presentation. The program requires a minimum of $330 \mathrm{~K}$ of RAM. The program executables, setup files, and documentation require $270 \mathrm{~K}$ of disk space. A data file for a 60 -stimulus test (typically $1 \mathrm{~h}$ in duration) occupies 2,100 bytes of disk space. The program utilizes a standard VGA graphics mode with a screen resolution of $640 \times 480$ pixels with 16 colors. All user inputs and responses are entered through the keyboard interface.

Several modifications have been made since the initial version (Fine et al., 1994) was created. (1) The graphics rendering method has been replaced so that it can run independently of monitor and video card combination. The graphics initialize in less than a second on a 486 machine; this took up to $30 \mathrm{sec}$ with the original software. (2) Subject and session information can now be entered from an initialization file or another program in addition to the original method of prompting the subject. This allows the program to be run from another program that presents a complete battery of tests. (3) Stimulus positions and delay times are no longer limited to six preset pseudo-random configurations. In addition, these parameters can be generated randomly at program initialization. (4) Testing parameters can easily be changed to any length of time and number of stimuli and are no longer limited to $10-\mathrm{min}$ blocks of 10 stimuli. (5) In addition to individually establishing brightness thresholds for each subject with a threshold exam, the experimenter can establish a fixed brightness value for all subjects. (6) A utility software routine will tabulate and analyze data from all subjects into a single file. (7) Reaction timing has been modified to avoid problems with keyboard buffer delays. A Pascal algorithm that polls at the system bios level (Crosbie, 1989) was adapted for the program.

\section{SETUP.EXE}

The setup program included with the SVT is a menudriven application that allows the experimenter to set up most features of the test. It will generate a SETUP.INI file that contains information about the number of stimuli, the total duration of the test, the dimensions of the stimulus, the background intensity, the default stimulus intensity, an optional target data directory, and optional comments. In addition, the file may contain an individual's stimulus level from the threshold exam for each subject. 
SETUP.INI is the default method to pass parameters to the SVT program.

The setup program queries the user about the method of determining time delays and stimulus positions. The SVT program can either calculate the delay to next stimulus and stimulus position randomly at the beginning of each test session, or it can read these values from a pseudorandom file. The setup program can generate nine time/ position files for such use. The average delay between stimuli is calculated from the total number of stimuli and the total duration of the exam. The minimum time delay is the average time delay divided by 3 , and the maximum is the average time delay multiplied by 3.5 (i.e., 10 stimuli in $10 \mathrm{~min}$ : The calculated average delay is $60 \mathrm{sec}$, the minimum delay is $20 \mathrm{sec}$, and the maximum is $210 \mathrm{sec}$ ).

The setup program also runs a threshold determination test automatically by running the SVT program with the correct parameters. The threshold test uses an adaptive algorithm to establish a threshold value that produces the chosen percentage correct value. Using the background level, and block dimensions from the SETUP.INI file along with a percent-correct goal specified by the experimenter, a threshold test is performed. The test provides a stimulus on the average of once every $12 \mathrm{sec}$. The brightness of the stimulus changes according to the prior performance of the subject. A value is chosen when the subject continuously attains the percent-correct goal for a given stimulus brightness level. The test usually takes between 7 and $10 \mathrm{~min}$ to perform. Typically on the first day of training, the subject performs a short threshold test designed to determine the gray shade of the stimulus at which $60 \%-80 \%$ of the stimuli are correctly detected. This performance level on the threshold test yields approximately $50 \%$ correct detection levels on a $2-\mathrm{h}$ test period with normal subjects (Fine et al., 1994). The chosen value is appended to the SETUP.INI file.

A subroutine to calibrate the brightness of the display is included in the setup program. This will either paint half the display the background shade and half the display the stimulus shade, or paint four blocks of predetermined shades. This allows the experimenter to use a light meter to calibrate the brightness and contrast on the monitor to match other monitors, or past tests.

\section{VISVIG.EXE}

Parameters for the SVT can be provided via a setup file or command-line parameters, or they can be prompted by the program. The program begins by prompting for the subject's name (or subject number) and then the session number. These are then combined with the extension .VIG to form the file name of the output data. The program then displays instructions to the subject and requires that the space bar be pressed to begin the test session. The area of the monitor in which the stimuli appear is defined as a circular area with a diameter equal to the width of the video monitor. During the course of the exam, the subject must scan the test area and press the space bar when a stimulus is detected. Finally, a short message announces the end of the test. If desired, the performance of the subject can be displayed on screen. The program is terminated either when the test time specified has elapsed or CTRL-Z is pressed. If CTRL-Z is used to terminate the program, an entry is made at the end of the data file.

Other options that can be executed include a feature to defeat screen savers; to present a tone whenever a subject responds; to present a tone if a key is held in the down position; to display a help screen; and to run practice tests (data are not saved).

An ASCII text output file is generated by the program with header information that includes the subject's name, session number, and all the values prompted for by the setup program. Data from the SVT are stored in columns. Each stimulus presentation is stored as the event occurs, to prevent data loss in case of system failure. The data stored include the time at which the stimulus has occurred, the number of cumulative correct detections, the stimulus number, the cumulative number of false alarms, the number of milliseconds before the subject pressed the space bar (zero if missed), and the Cartesian coordinates of the stimulus.

\section{ANALYZE.EXE}

The Analyze program included with SVT is a menudriven application that allows the experimenter to calculate a number of statistical indices. The Analyze option will work for selected. VIG files, or all .VIG files present. The statistics computed by the program can be selected by the experimenter and can include the subject's name, the total number of stimuli, the session number, the total number of hits, the total number of misses, the total number of false positives, the mean response time, the standard deviation of the response time, the standard error of mean of the response time, the time, the date, the average delay between stimuli, comments ( 8 characters), the longest sequence of missed stimuli, the largest number of false positives between stimuli, and the longest sequence of correct responses. Output is written in an ASCII format with the column separator of choice (tab, space, comma, or colon).

\section{AVAILABILITY}

The compiled programs and a detailed set of instructions in an ASCII text file are available on written request to the first author. To obtain them, forward a formatted 3.5in. floppy disk and a stamped, self-addressed envelope suitable for return mailing of the disk.

\section{REFERENCES}

Clubley, M., Bye, C. E., Henson, T. A., Peck, A. W., \& Ridnington, C. J. (1979). Effects of caffeine and cyclizine alone and in combination on human performance, subjective effects and EEG activity. British Journal of Clinical Pharmacology, 7, 157-163.

Craig, A., Wilkinson, R. T., \& Colquhoun, W. P. (1981). Diurnal variation in vigilance efficiency. Ergonomics, 24, 641-651. 
Crosbie, J. ( 1989). A simple Turbo Pascal 4.0 program for millisecond timing on the IBM PC/XT/AT. Behavior Research Methods, Instruments, \& Computers, 21, 408-413.

Dinges, D. F. (1992). Probing the limits of functional capability: The effects of sleep loss on short-duration tasks. In R. J. Broughton \& R. Ogilvie (Eds.), Sleep, arousal and performance (pp. 176-188) Cambridge, MA: Birkhauser.

Dollins, A. B., Lynch, H. J., Deng, M. H., Kischka, K. U., Gleason, R. E., Wurtman, R. J., \& Lieberman, H. R. (1993). Effect of pharmacological daytime doses of melatonin on human mood and performance. Psychopharmacology, 112, 490-496.

Dollins, A. B., Lynch, H. J., Wurtman, R. J., Deng, M. H., \& LieberMAN, H. R. (1993). Effects of illumination on human nocturnal serum melatonin levels and performance. Physiology \& Behavior, 53, 153 160.

Fine, B. J., Kobrick, J. L., Lieberman, H. R., Marlowe, B., Riley, R. H., \& Tharion, W. J. (1994). Effects of caffeine or diphenhydramine on visual vigilance. Psychopharmacology, 114, 233-238.

Hirshoowitz, M., De La Cueva, L., \& Herman, J. H. (1993). The multiple vigilance test. Behavior Research Methods, Instruments, \& Computers, 25, 272-275.

KoELEGA, H. S. (1989). Benzodiazepines and vigilance performance: A review. Psychopharmacology, 98, 146-156.

Lieberman, H. R. (1989). Cognitive effects of various food constituents. In R. Shepherd (Ed.), Handbook of the psychophysiology of human eating (pp. 251-270). Chichester, U.K.: Wiley.

Lieberman, H. R., Wurtman, R. J., Garfield, G. S., Roberts, C. H., \& Coviella, I. L. (1987). The effects of low doses of caffeine on human performance and mood. Psychopharmacology, 92, 308-312.

LiNNOILA, M. (1973). Effects of antihistamines, cholormezanone and al- cohol on psychomotor skills related to driving. European Journal of Clinical Pharmacology, 5, 247-254.

MACKIE, R. R. (1987). Vigilance research-Are we ready for countermeasures? Human Factors, 29, 707-723.

MitLER, M. M. (1988). Catastrophes, sleep and public policy: Consensus report. Sleep, 11, 100-109.

Office of TeChNology AsSessment, U.S. Congress (1991). Biological rhythms: Implications for the worker (OTA-BA-463). Washington, DC: U.S. Government Printing Office.

Ogilvie, R., \& Broughton, R. J. (1992). Bibliography of Robert T. Wilkinson: A commentary. In R. J. Broughton \& R. Ogilvie (Eds.), Sleep, arousal and performance (pp. 266-273). Cambridge, MA: Birkhauser.

TiPlaDy, B. (1992). Continuous attention: Rationale and discriminant validation of a test designed for use in psychopharmacology. Behovior Research Methods, Instruments, \& Computers, 24, 16-21.

Wiener, E. L. (1988). Cockpit automation. In E. L. Wiener \& D. C. Nagel (Eds.), Human factors in aviation (pp. 433-461). San Diego: Academic Press.

Wilkinson, R. T. (1968). Sleep deprivation: Performance tests for partial and selective sleep deprivation. In B. F. Reiss \& L. A. Abt (Eds.), Progress in clinical psychology (pp. 28-43). New York: Grune \& Stratton.

WILKINSON, R. T. (1992). The measurement of sleepiness. In R. J. Broughton \& R. Ogilvie (Eds.), Sleep, arousal and performance (pp. 255-265). Cambridge, MA: Birkhauser.

(Manuscript received February 9, 1996; revision accepted for publication April 28, 1997.) 